\title{
Swelling Equilibrium and the Superstructures of Uniaxially Oriented $\propto$ Nylon 6 in Solvent Mixtures
}

\author{
Zeyong Zheng, Shuichi NoJima, Takashi YAMANE, \\ and Tamaichi AsHIDA \\ Department of Applied Chemistry, Faculty of Engineering, \\ Nagoya University, Nagoya 464-01, Japan
}

(Received May 9, 1988)

\begin{abstract}
A small angle X-ray scattering (SAXS) study of uniaxially oriented $\alpha$ nylon 6 at swelling equilibrium was carried out based on a paracrystalline macrolattice model. The results supported the conventional assumption that the crystalline core is unpenetrable to the swelling agent. Reasonable thermodynamic interaction parameters were obtained, and excellent agreement between the dimensions of the tie molecules from the thermodynamic experiment and SAXS data was obtained, indicating that the tie molecules were highly extended at maximum swelling degree. KEY WORDS Swelling Equilibrium / Three-Component System / Interaction Parameter / $\alpha$ Nylon 6 / Small Angle X-Ray Scattering / SAXS Model Fitting / Crystallite /
\end{abstract}

The swelling behavior of the semicrystalline polymers has been investigated ${ }^{1-10}$ using the SAXS method. In all these investigations, however, discussions have exclusively been confined to the change in the long spacing, using only a portion of the information contained in the SAXS pattern. Recently, a model which accounts for SAXS pattern from uniaxially oriented semicrystalline polymers has been proposed by Fronk and Wilke et al. ${ }^{11-13}$ This model makes it possible to clarify the behavior of the crystallites in polymers when swollen, and to examine the validity of the conventional assumption that the crystalline phase is unpenetrable to swelling agent. The assumption was first introduced by Rogers et al. ${ }^{14}$ and adopted by many other authors ${ }^{15-19}$ to apply the theor ${ }^{20}$ of swelling equilibrium for crosslinked rubber to semicrystalline polymer.

In this work, the SAXS study of uniaxially oriented $\alpha$ nylon 6 at swelling equilibrium was made, and the superstructure parameters were obtained using the above model. To make clear the dependence of the superstructure on the degree of swelling, measurements were performed for a series of mixtures of the solvent (swelling agent) with nonsolvent of different concentrations, hence different solvent activities. The nonsolvent was chosen on the basis of its lack of affinity for the polymer and miscibility with the solvent. The thermodynamic treatment was attempted to shed light on how the swelling theory is applicable to the present three-component, polymer-solventnonsolvent systems.

For nylon 6 a large number of studies ${ }^{21-32}$ has been conducted on the effects of swelling treatment on the morphology and the properties, but no report deals with the structural behavior at swelling equilibrium.

\section{THEORY}

Thermodynamic Treatment of Polymer-SolventNonsolvent Three-Component System

It has been shown ${ }^{33-35}$ that in the case of the sorption of solvent by a polymer from the mixture of solvent and nonsolvent having no 
affinity for the polymer, the nonsolvent plays merely the role of an inert diluent and may not enter the polymer. In order to apply the FloryHuggins-Rehner theory ${ }^{20}$ to a semicrystalline polymer, Rogers et al. ${ }^{14}$ introduced the assumptions that crystallites play a role of chemical crosslinks in the case of rubber and swelling occurs only in the amorphous phase. Brown ${ }^{18}$ modified the theory by making allowance for the fact that the crystallites (lamellar texture) restrict the swelling in the lateral direction, leading to the expression for $\Delta \mu_{1}^{P}$, the difference between the chemical potential of the solvent in the swollen polymer and that in the pure state

$$
\begin{aligned}
\Delta \mu_{1}^{P} & =\ln \left(1-\varphi_{2 a}\right)+\varphi_{2 a}+\chi \varphi_{2 a}^{2}+\frac{\rho_{a} V_{1}}{M_{c}} \varphi_{2 a}^{-1} \\
& =\ln \left(1-\varphi_{2 a}\right)+\varphi_{2 a}+\chi_{1} \varphi_{2 a}^{2}
\end{aligned}
$$

where $\chi$ is the polymer-solvent interaction parameter, $\rho_{a}$ density of the amorphous phase before swelling, $M_{c}$ the molecular weight of the polymer chain lying in the amorphous region between crystallite "crosslinks", $V_{1}$ the molar volume of the solvent, $\varphi_{2 a}$ the volume fraction of polymer in the swollen amorphous phase and $\chi_{1}$ the effective interaction parameter defined by

$$
\chi_{1}=\chi+\frac{\rho_{a} V_{1}}{M_{c}} \varphi_{2 a}^{-3}
$$

The difference between the chemical potential of the solvent in the ambient solution and that in the pure state $\Delta \mu_{1}^{S}$, or solvent activity can be expressed as

$$
\Delta \mu_{1}^{S}=R T\left\{\chi_{1 N}\left(1-y_{1}\right)^{2}+\ln y_{1}\right\}
$$

where $y_{1}$ is the molar fraction of solvent in the ambient solution, and $\chi_{1 N}$ the solventnonsolvent interaction parameter.

The thermodynamical condition of the swelling equilibrium in a three-component system can be written as

$$
\Delta \mu_{1}^{P}=\Delta \mu_{1}^{S}
$$

Inserting eq 1 and 4 into 5 gives the final thermodynamic expression for the threecomponent system.

$$
\begin{gathered}
\ln \left(1-\varphi_{2 a}\right)+\varphi_{2 a}+\chi \varphi_{2 a}^{2}+\frac{\rho_{a} V_{1}}{M_{c}} \varphi_{2 a}^{-1} \\
=\chi_{1 N}\left(1-y_{1}\right)^{2}+\ln y_{1}
\end{gathered}
$$

Apparently, three unknown parameters, $\chi$, $M_{c}$, and $\chi_{1 N}$ are contained in the equation. Therefore, as long as the swelling degrees corresponding to at least three solvent activities $\left(y_{1}\right)$ are measured, the above parameters can be independently determined.

Because the saturated vapor pressures of both benzyl alcohol and phenol used as active solvents in this study are extremely small at room temperature $(<1 \mathrm{mmHg})$, it is almost impossible to determine $\chi$ and $M_{c}$ with the usual method based on vapor pressure. ${ }^{14-19}$ With our method described above (eq 6), however, these parameters can be easily determined without sophisticated apparatus.

\section{Model Calculation of SAXS Intensity}

A general intensity formula of the small angle X-ray scattering from polymers with uniaxial orientation has recently been derived by Fronk and Wilke, ${ }^{12}$ based on the assumptions:

(1) It is possible to define a structural perpendicular $\boldsymbol{n}$ for the basic scattering unit (cluster);

(2) The cluster orientation shows rotational symmetry about $\boldsymbol{n}$;

(3) The distribution of the structural perpendiculars $\boldsymbol{n}$ is given by a function with rotational symmetry about the fiber axis $f$ (draw direction), and the orientation with respect to $f$ is defined by the distribution density $D(\alpha)$, with $\alpha$ being the angle between $\boldsymbol{n}$ and $\boldsymbol{f}$.

Under these assumptions, the scattered intensity is given as ${ }^{11-13}$

$$
I\left(r^{*}, \theta^{*}\right)=\sum_{n=0}^{\infty}\left\{\frac{2}{4 n+1} D_{2 n} G_{2 n}^{0}\left(r^{*}\right) P_{2 n}\left(\cos \theta^{*}\right)\right\}
$$


where

$$
\begin{gathered}
D_{2 n}=\frac{4 n+1}{2} \int_{0}^{\pi} \sin \alpha D(\alpha) P_{2 n}(\cos \alpha) \mathrm{d} \alpha \\
G_{2 n}^{0}\left(r^{*}\right)=\frac{4 n+1}{4} \int_{0}^{\pi} \sin \psi^{*} P_{2 n}\left(\cos \psi^{*}\right) \mathrm{d} \psi^{*} \\
\quad \times \int_{0}^{2 \pi} I_{1}(\boldsymbol{b}) \mathrm{d} \varphi^{*}
\end{gathered}
$$

where $r^{*}, 0^{*}\left(\psi^{*}\right)$, and $\varphi^{*}$ are spherical coordinates in the reciprocal space, $\boldsymbol{b}$ the scattering vector, $P_{2 n}$ Legendre polynomials, and $I_{1}(b)$ the scattered intensity of a single basic unit proportional to the squared absolute value of the Fourier transform of the cluster density $\rho(\boldsymbol{r})$. Details of the derivation of eq $7-9$ are given in ref $11-12$.

As in the case of highly oriented polyethylene, ${ }^{13}$ the distribution density $D(\alpha)$ in eq 8 for highly uniaxially oriented $\alpha$ nylon 6 can be assumed to be a Gaussian function centered at $\alpha=0$.

$$
D(\alpha)=\frac{1}{\sqrt{2 \pi} \sigma} \exp \left\{-\frac{1}{2}\left(\frac{\alpha}{\sigma}\right)^{2}\right\}
$$

The basic scattering unit is assumed to be a three-dimensional, paracrystalline monoclinic macrolattice composed of identical crystallites. The scatteredintensity $I_{1}(\boldsymbol{b})$ in eq 9 from the unit is given $b^{36}$

$$
I_{1}(\boldsymbol{b})=|F(\boldsymbol{b})|^{2} Z(\boldsymbol{b})
$$

where $F(b)$ is the scattered amplitude of a single crystallite and $Z(b)$ is the lattice factor of an ideal paracrystalline macrolattice. ${ }^{37}$ Both $F(b)$ and $Z(b)$ are functions of the superstructure parameters ${ }^{12,13,36}$ as tabulated in Table II, where the nomenclature conforms to those used in ref 12 .

\section{EXPERIMENTAL}

\section{Preparation of Samples}

Nylon 6 used in this study was supplied by Toray Industry Inc. in the form of an un- oriented sheet with a thickness of $0.5 \mathrm{~mm}$. Uniaxially oriented $\alpha$ nylon 6 for a subsequent swelling experiment was prepared in three steps: (1) Three strips each $30 \mathrm{~mm} \times 3 \mathrm{~mm}$ were cut out from the original sheet, joined together with $85 \%$ aqueous formic acid solution, and drawn with necking to a natural draw-ratio of 3 at the draw-rate of $100 \mathrm{~mm} / \mathrm{min}$ at room temperature (cold-drawing). It was confirmed by wide angle X-ray scattering (WAXS) that the crystallites in the resultant sample consist of $\alpha, \beta$, and $\gamma$ forms. (2) The samples were annealed at elevated temperature for $3 \mathrm{~h}$ at constant length. It is well known that the major part of $\beta$ and $\gamma$ forms is transformed into the $\alpha$ form by annealing above $185^{\circ} \mathrm{C}$, and also that the superstructure of nylon 6 is significantly influenced by the annealing temperature ranging from $190 \mathrm{C}$ to the melting temperature. Two annealing temperatures, $190^{\circ} \mathrm{C}$ (sample A) and 196 C (sample B), were chosen to investigate the effects of the thermal history on swelling behavior. (3) In order to remove traces of the $\beta$ and $\gamma$ forms, the sample was further treated with $0.4 \%$ aqueous phenol solution for 4 days. Finally, the sample was washed in running water for one week to remove phenol from the sample. The results of WAXS and SAXS showed that the crystallites with $\alpha$ form were uniaxially oriented in the sample.

\section{Swelling Experiment}

A swelling experiment was carried out for four three-component, polymer-solventnonsolvent systems: a, sample A-benzyl alcohol-cyclohexanone; b, sample B-benzyl alcohol-ethyl acetate; c, sample B-benzyl alcohol-cyclohexanone; d, sample B-phenol$n$-heptane. Benzyl alcohol can penetrate nylon 6 but never dissolves at room temperature. Thus, the solvent composition, or weight fraction of the solvent in the solvent-nonsolvent mixture, was changed to the range from 0.2 to 1.0 for systems $a, b$, and $c$. Phenol, on the other hand, is a good solvent for nylon 6; thus, 
the swelling experiment was done at low composition ranging from 0.002 to 0.010 for the system d. Equilibrium swelling degrees $Q$, defined as $\left(W-W_{0}\right) / W_{0}$ with $W_{0}$ and $W$ being the weights of the dry and swollen samples, were measured at $27 \mathrm{C}$ for various solvent compositions. It took 2 to 3 days to reach equilibrium for all samples investigated.

\section{SAXS Measurements}

SAXS data were obtained with a onedimensional position sensitive proportional counter (PSPC) using a rotating anode X-ray generator (RU300, Rigaku) operating at $50 \mathrm{kV}$ and $80 \mathrm{~mA}$. The SAXS optics had a three pinhole $(0.5,0.3$, and $0.5 \mathrm{~mm}$ in diameter) collimation system, with which Bragg spacings up to $250 \AA$ could be completely resolved from the direct beam. The intensity profiles along

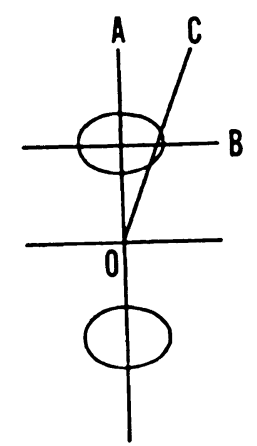

Figure 1. Schematic representation of a two-point diagram. the three sections $\mathrm{A}, \mathrm{B}$, and $\mathrm{C}$ (Figure 1) were measured.

Section A: through the origin and along the meridian;

Section B: parallel to the equator, and through the maximum of intensity;

Section $C$ : through the origin and forming an angle of 20 with the meridian.

The measurements were performed by rotating the sample in the plane perpendicular to the incident beam or moving PSPC in the meridional direction. The sample for SAXS measurement was held in a cell with enough ambient solvent which keeps the sample at swelling equilibrium. A counting time of $600 \mathrm{~s}$ was sufficient for each section. Corrections for the PSPC sensitivity and linearity, sample absorption, and background scattering were made, and the measured intensity was smoothed using the stepwise cubic least-squares routine to minimize the statistical error.

\section{Analysis of SAXS Intensity}

The parameters characterizing the superstructure of the sample, as shown in Table II, were evaluated by fitting the theoretical intensity calculated with eq 7 to the experimental one using the least-squares routine SALS with the Gauss-Newton algorithm. To reduce the calculation time, ${ }^{12.13}$ only 6 - 10 points were considered for each section during the fitting. An example of the best fit is shown in Figure 2,
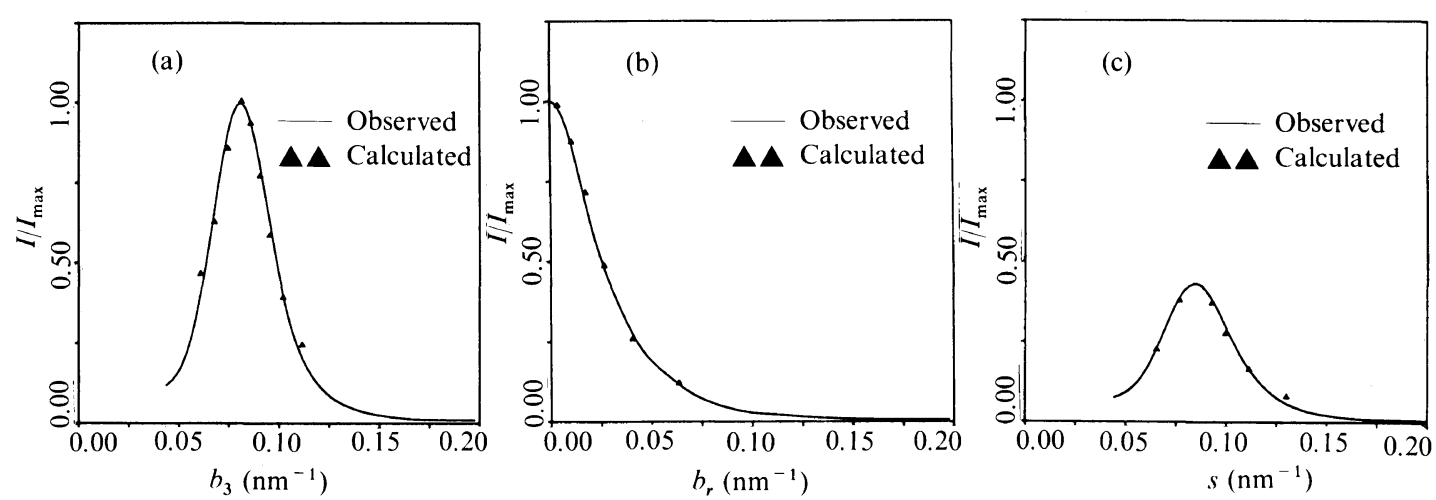

Figure 2. An example of the best fit between calculated and observed SAXS intensities. (a) section A; (b) section $\mathrm{B}$; (c) section $\mathrm{C}$. 
where the experimental intensities are given as curves and the calculated ones discrete points since only a small number of the intensity data was used in the model fitting.

\section{RESULTS}

\section{Swelling}

The swelling degrees for the four systems are shown in Figure 3. The solvent concentration in the mixture after equilibrium sorption is assumed to be equal to the starting one for systems a, b, and c, whereas it was corrected for $d$ taking into account that the amount of solvent absorbed by polymer could no longer be neglected as compared to that remaining in the ambient solution.

Fitting the observed swelling degrees shown in Figure 3 to the theoretical equation (eq 6) was completed with a linear least-squares routine. Plausible fits were obtained for systems a and $\mathrm{d}$, but attempts to achieve reasonable fits for systems $\mathrm{b}$ and $\mathrm{c}$ failed. The least-squares results of the parameters $M_{c}, \chi$, and $\chi_{1 N}$ are listed in Table I. For d, the direct use of observed data in fitting could not be made because of the insufficient number and quality of data. Therefore, the fitting was carried out on the smoothed plot in Figure $3 b$.

According to the modified theory (eq 2 and
$3)$, the effective interaction parameter $\chi_{1}$ is linearly related to $\varphi_{2 a}^{-3}$. The plots for a and $\mathrm{d}$ are shown in Figure 4 , where the intercept gives $\chi$ and the slope $\rho_{a} V_{1} / M_{c}$.

\section{SAXS Data}

Both samples $A$ and $B$ exhibit typical twopoint diagrams (Figure 5), though their peak positions and widths are different from each other. The SAXS profiles from system a with solvent concentrations of 20,80 , and $100 \%$ $(\mathrm{w} / \mathrm{w})$ are given in Figure 6, and those along section A of system d in Figure 7, where the intensities are plotted against reciprocal coordinates $\left(b_{r}=r^{*} \sin \theta^{*}, b_{3}=r^{*} \cos \theta^{*}\right.$, and $s=$ $\left.r^{*}\right)$ for convenience to compare with the theoretical ones calculated with Wilke's model. The SAXS data of systems $b$ and $c$ are similar

Table I. Interaction parameters and molecular weights $M_{c}$ of sample A (system a) and sample B (system d)

\begin{tabular}{lcc}
\hline & Sample A & Sample B \\
\hline$M_{c}$ & 790 & 787 \\
$\chi$ & $0.51^{\mathrm{a}}$ & $-0.10^{\mathrm{b}}$ \\
$\chi_{1 N}$ & $0.38^{\mathrm{c}}$ & $4.83^{\mathrm{d}}$ \\
\hline
\end{tabular}

\footnotetext{
${ }^{\text {a }}$ Interaction parameter between $\alpha$ nylon 6 and benzyl alcohol.

b That between $\alpha$ nylon 6 and phenol.

c That between benzyl alcohol and cyclohexanone.

d That between phenol and $n$-heptane.
}
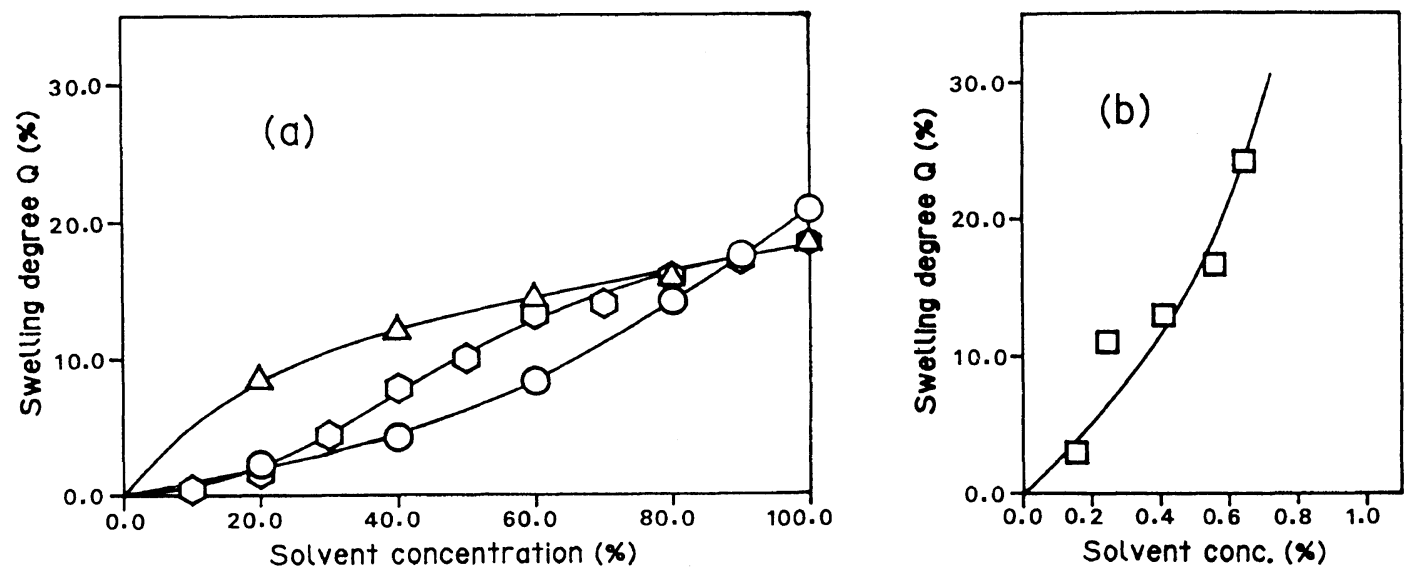

Figure 3. Dependence of the swelling degree $Q$ on solvent concentration. (a) for systems a $(\bigcirc), \mathrm{b}(\triangle)$, and c (O); (b) for system d ( $\square$ ). 
to those of the other systems.

As apparent in Figures 6 and 7, SAXS patterns along section A from the swollen samples shift toward the lower angle side with

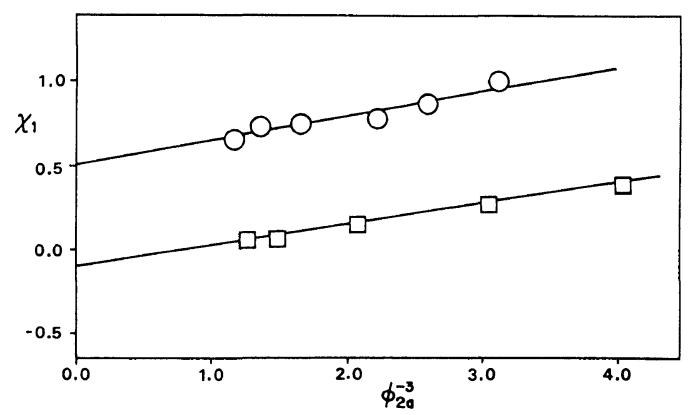

Figure 4. Least-squares plots of $\chi_{1}$ vs. $\varphi_{2 a}^{-3}(\bigcirc$ for system a; $\square$ for d). increasing swelling degree $Q$, corresponding to the increase in the long spacings. Another feature is the reversible sharpening of the peak with increasing $Q$ both for sections A and B. The plot of half height widths $v s$. long spacings is given in Figure 8. In addition to changes in the peak position and width, the pronounced change in the SAXS profiles before and after swelling was observed at the lower angle side. Before swelling they had additional strong diffuse scattering, which attenuated rapidly with increasing scattering angle, while those of the swollen samples had no such scattering (comparing Figures 5 and 7). This change was found to be roughly reversible since the scattering reappeared when the solvent was removed, with a little increase in intensity.
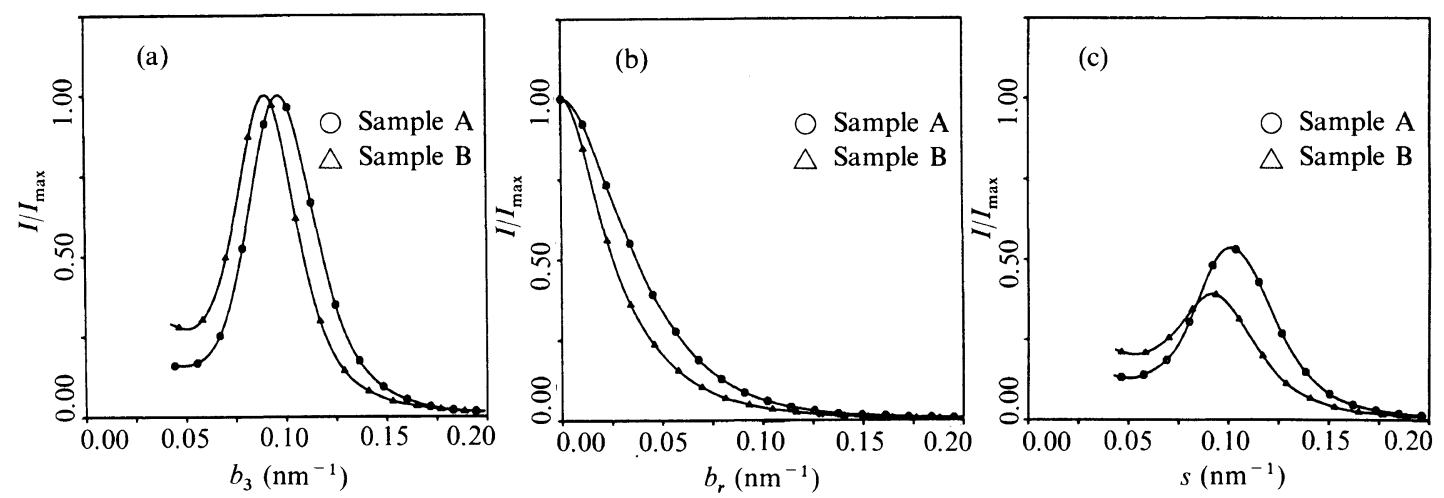

Figure 5. The observed SAXS profiles of unswollen samples A and B. (a) section A; (b) section B; (c) section $\mathrm{C}$.
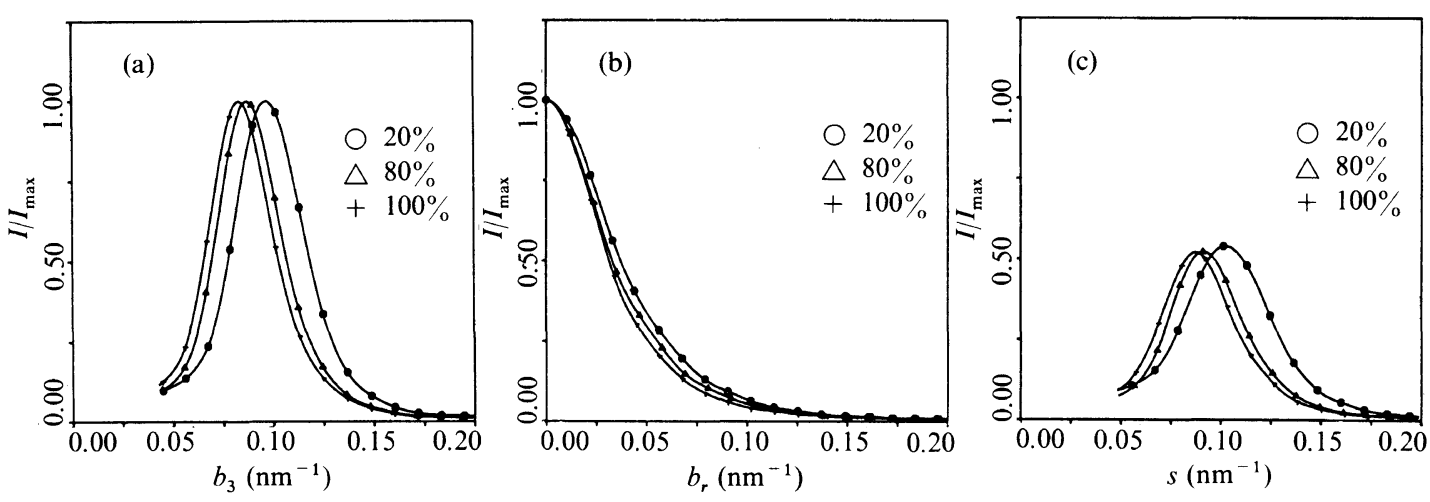

Figure 6. The observed SAXS profiles of the samples in system a (sample A) at swelling equilibrium. (a) section A; (b) section B; (c) section $\mathrm{C}$. The numbers are the percentages of solvent in the mixtures with nonsolvent. 


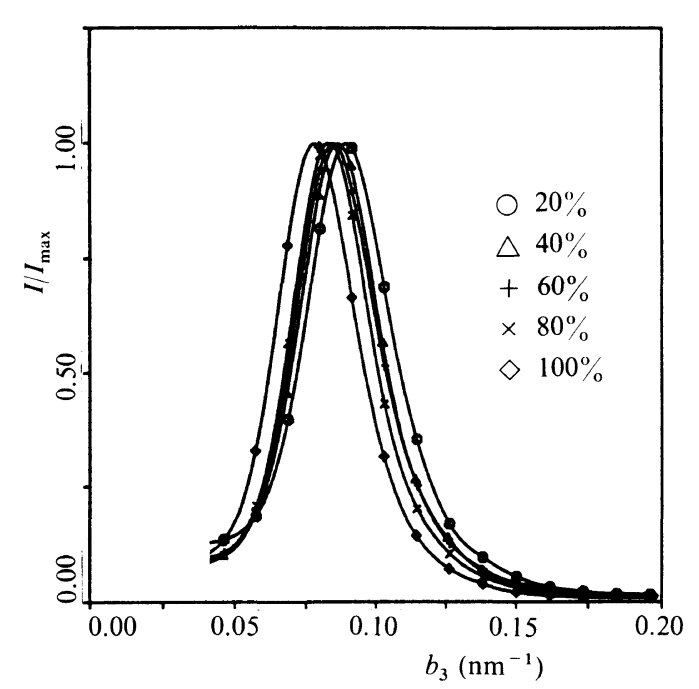

Figure 7. The observed SAXS profiles along section A of the samples in system $d$ (sample B) at swelling equilibrium. The numbers are the percentages of solvent in the mixtures with nonsolvent.

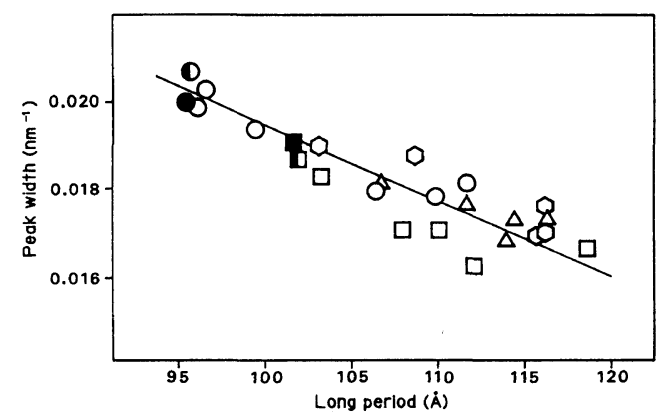

Figure 8. Dependence of the peak widths of section $\mathrm{A}$ on the long period $a_{3}(O$, for system a; $\triangle$, for $\mathrm{b} ; O$, for c; $\square$, for d; , $\square$, before swelling; $\boldsymbol{\top}, \square$, after removing the solvent).

\section{DISCUSSION}

\section{Swelling Equilibrium}

Equilibrium Degrees of Swelling. Comparing the plots of systems $b$ and $c$ in Figure $3 a$, it is apparent that the equilibrium swelling degrees are significantly dependent on the nature of the nonsolvent at a low solvent concentration, while independent at a high one, indicating that the interaction between the solvent and nonsolvent influences appreciably the solvent activity at the former, while not at the latter.
This may be explained in terms of the variation in the disposition of the solvent and nonsolvent. At low concentration the solvent mixture may be pictured as solvent molecules being encircled by nonsolvent molecules, so the solvent has to overcome the strong interaction with nonsolvent so as to migrate to the polymer surface and enter it. In such a situation, the solvent activity is determined by the contribution of enthalpy as well as that of entropy. On the contrary, at high concentration the disposition of solvent and nonsolvent is reversed. The contribution of enthalpy is no longer prominent, and the solvent activity is determined primarily by the contribution of entropy.

Interaction parameters. The interaction parameters of nylon 6 with benzyl alcohol and phenol are unknown, so we can not make a direct comparison with results based on other techniques. However, as compared to the results for high-density polyethylene and polypropylene obtained by many authors, ${ }^{14,16-18}$ our results seem to be quite reasonable, both for $M_{c}$ and interaction parameters. The relatively large $\chi, 0.51$ (see Table I) for the interaction of $\alpha$ nylon 6-benzyl alcohol falls in the general (positive) range of $\chi$ measured for amorphous polymer-solvent systems; ${ }^{38-40}$ and the small minus $\chi,-0.10$ of system $\mathrm{d}$ also seems to be plausible since phenol has very strong solvating power for nylon 6 .

The interaction parameters between solvent and nonsolvent also appear reasonable. The large value of $\chi_{1 N}, 4.83$ for phenol- $n$-heptane interaction accounts for the difficulty for phenol to dissolve in $n$-heptane. The interaction parameter between benzyl alcohol and ethyl acetate (system b) could not be estimated, but it must assume a value larger than that, 0.38 , between benzyl alcohol and cyclohexanone, based on the observation that solvent activity is larger in a mixture with ethyl acetate than in that with cyclohexanone (Figure 3a).

Molecular weight $M_{\mathrm{c}}$. In addition to the interaction parameters, reasonable values of 
$M_{c}$ were obtained both for system a (sample A) and system d (sample B) (see Table I). Surprisingly, the two $M_{c}$ 's are so close that one can consider them to be the same, and correspond to about 7 repeating units. Information relevant to $M_{c}$ is also available from the following SAXS analysis.

The reasonable values of $\chi, \chi_{1 N}$, and $M_{c}$ thus obtained imply in turn the validity of the swelling equilibrium description in terms of eq 6 for the present three-component systems.

\section{SAXS Analysis}

Peak intensity. The observed peak intensities were plotted against the swelling degree in Figure 9. They were observed to increase linearly with the swelling degree for all systems studied. The increase in peak intensity after swelling was also observed for polyethylene ${ }^{3}$ and doubly oriented nylon 11. ${ }^{1}$ Dosiere and Point ${ }^{1}$ explained the increase by estimating the variation of the mean-square electron density fluctuations $\left\langle\Delta \eta^{2}\right\rangle_{s}$ due to swelling. It is not surprising that the peak intensities of systems a and $b$ are the same because samples $A$ and $B$ have very close crystallinities, 0.51 and 0.53 respectively based on density. The peak intensity of $d$ shows similar trend but larger than the others. This is because the electron densities of benzyl alcohol, $0.559 \mathrm{~mol} \mathrm{el} \mathrm{cm}^{-3}$ and phenol, $0.558 \mathrm{~mol} \mathrm{el} \mathrm{cm}^{-3}$ are almost the same and the crystallinity increased from 0.53 for the samples of system b to 0.58 for $d$.

Microvoids. The scattering at the lower angle can be explained by the existence of microvoids in the amorphous phase. That is, the diffuse scattering is ascribed to the electron density difference between microvoids and surrounding materials. On swelling, such difference was lost because the voids had either merely been filled with solvent having electron density closer to that of the amorphous phase, or disappeared to become a homogeneous swollen amorphous phase contrasting with the crystalline phase. Regeneration of microvoids after removal of the solvent is a matter of

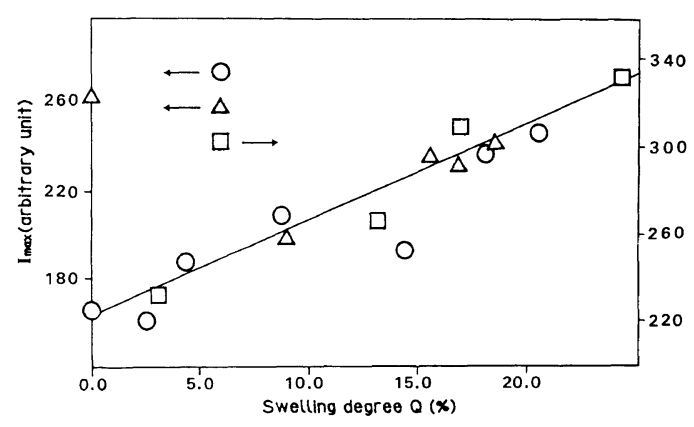

Figure 9. Peak intensity $v s$. swelling degree. The symbols are the same as in Figure 8.

course in the former case; and in the latter case, it also seems to be possible because they were originally generated by aqueous phenol treatment, before which no such scattering was observed. We expect that systems $\mathrm{b}$ and $\mathrm{c}$ follow the former mechanism and system $d$, the latter. This distinction, if true, may be the reason why the swelling behavior of system $d$ can be described by eq 6 but those of $b$ and $c$ cannot.

Other evidence supporting the existence of microvoids is the change of SAXS peak intensity. The intensity of unswollen sample B (Figure 9) deviating significantly from the extrapolated value implies that there exists an additional contribution of microvoids to electron density fluctuation between the crystalline phase and surrounding substances.

In contrast with other systems, the extrapolated value of the peak intensity of system a agrees well with that of the dry sample. Therefore, we believe that the microvoid content in sample $\mathrm{A}$ is negligible compared to that in sample B.

Superstructures before Swelling. The superstructure parameters of samples A and B before swelling are listed in Table II. The thickness of the amorphous phase in sample $A$ is $47 \AA$, and that of sample B $45 \AA$ (Table II). The two values are very close to each other, which corresponds to the thermodynamical observation that the tie molecular weights $M_{c}$ 's of the two samples are the same, and at the 
Table II. The superstructure parameters of samples $\mathrm{A}$ and $\mathrm{B}$ before swelling

\begin{tabular}{lcc}
\hline & Sample A & Sample B \\
\hline$a_{3}(\AA)$ & 95.4 & 101.2 \\
$a_{r}(\AA)$ & 174.2 & 181.4 \\
$D_{1}(\AA)$ & 140.5 & 156.3 \\
$D_{2}(\AA)$ & 76.1 & 73.2 \\
$H(\AA)$ & 48.5 & 56.4 \\
$a_{3}-H(\AA)^{*}$ & 46.9 & 44.8 \\
$\beta\left({ }^{\circ}\right)^{* *}$ & 90.0 & 90.0 \\
$g_{3}$ & 0.024 & 0.029 \\
$g_{r}$ & 0.384 & 0.225 \\
$g_{33}$ & 0.259 & 0.262 \\
$\sigma\left({ }^{\circ}\right)^{* *}$ & 12.5 & 12.5 \\
\hline
\end{tabular}

* Thickness of the amorphous layer.

** Fixed during fitting.

same time indicates that annealing at higher temperature increases the thickness of the lamella while leaving that of the amorphous phase unchanged. As can be seen in Table II, the value of $D_{1}$, the lateral dimension of the crystallites along the direction of the hydrogen bonds, ${ }^{41}$ is much greater for sample B than for sample $\mathrm{A}$, while $D_{2}$, dimension in the direction normal to the 001 plane containing the hydrogen bonds, ${ }^{41}$ exhibits no significant change, indicating more rapid growth of crystallites along the hydrogen bonds. ${ }^{41-42}$ These values well compare with those obtained by other techniques. ${ }^{41-43}$

Superstructures at Swelling Equilibrium. Superstructure parameters for system a are plotted in Figure 10 against swelling degree. No significant change was found in the crystallite thickness $H$ (Figure 10a); a slight increase in the lateral dimension $D_{1}$ along hydrogen bonds was accompanied by a corresponding decrease in their lateral distance $a_{r}$ between crystallites at low swelling degree (Figure 10b). Both $D_{1}$ and $a_{r}$ were almost invariable at the swelling degrees greater than $5 \%$. The behavior of the long periods $a_{3}$ with $Q$ can be divided into three ranges: at low $Q(<8 \%)$ $a_{3}$ increased moderately with increasing $Q$; at $8 \%<Q<16 \%, a_{3}$ increased rapidly; at
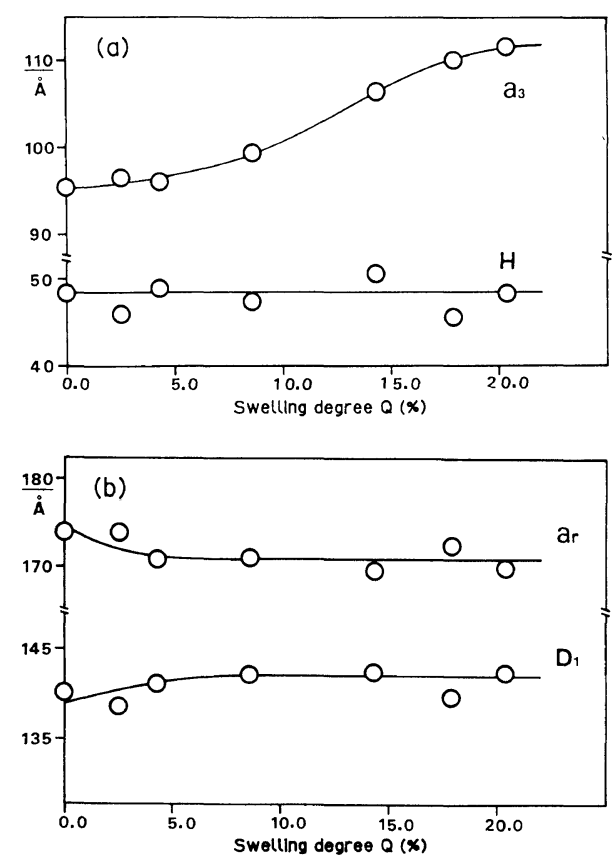

Figure 10. Dependence of the superstructure parameters of system a (sample A) on the swelling degree $Q$. (a) $a_{3}$ and $H$ vs. $Q$; (b) $a_{r}$ and $D_{1}$ vs. $Q$.

$Q>16 \%$, the increase slowed down again until $Q$ reached the maximum value (Figure 10a). The constant lateral distance $a_{r}$ implies that the crystallites are closely packed in the lateral direction. Without high affinity for the polymer, a solvent, such as benzyl alcohol in the present case, will not swell the materials in this direction, leading to remarkable anisotropy of swelling. ${ }^{1}$ The constancy of both $H$ and $D_{1}$ verifies that the solvent cannot penetrate the crystalline phase, which is requisite for application of the swelling theory to semicrystalline polymers.

The crystallite thickness $H$ of systems b, c, and d (Figure 11a) exhibits behavior different from that of system a. $H$ decreased moderately with increasing $Q$ and reached a minimum value at $Q \simeq 12.5 \%$, then increased with increasing $Q$, and finally became constant when $Q>16 \%$. Such behavior was found reversible. One cannot expect a true increase or decrease in thickness to be reversible with 
respect to the swelling degree. In order to explain this, we suppose that there exists a pseudocrystalline region outside the crystalline core. This region is made up of the molecular chain issuing from the core and entering amorphous phase without re-entry to become tie molecules and is penetrable to the solvent. Before swelling, the region acts as a crystalline phase. When moderately swollen, it is partly penetrated and disordered by the solvent and thus becomes thinner. As swelling increases, however, the tie-molecules become highly stretched (indicated by the rapid increase in $a_{3}$, see Figures $10 \mathrm{a}$ and $11 \mathrm{a}$ ). This allows no further disordering to occur in the region, resulting in minimum $H$ (Figure 11a). Further increasing swelling enhances the degree of chain extension, and thereby improves the "ordering" of the above region, the crystallites behaving as though it is growing. In sample A, there may be no such region, so the above behavior was not observed for system a.

Figure $11 \mathrm{~b}$ gives a plot of the lateral dimension $D_{1} v s$. swelling degree $Q$ for systems b, c, and $\mathrm{d}$. It is clear that up to the swelling degree of $18 \%, D_{1}$ was kept constant, whereas further increasing $Q, D_{1}$ began to decrease. This observation implies that as compared to the lamellar surface, the lateral surface is more subjected to the solvent because $H$ remained unchanged even at larger $Q$ (see Figure 11a). Thus, it is believed that if the solvent activity becomes large enough, dissolving will occur first in the lateral surface.

The behavior of $a_{3}$ of systems $\mathrm{b}$ and $\mathrm{c}$ (Figure 1la) was very similar to that of a (Figure 10a), while that of system $d$ was not. It increased almost linearly with increasing $Q$ (Figure 11c). Another characteristic difference between the behavior of system $d$ and the others was the change of $a_{r}$ (Figure $11 \mathrm{c}$ ). It varied approximately linearly with $Q$, but was constant for all other systems in this study (Figures $10 \mathrm{~b}$ and $11 \mathrm{~b}$ ), reflecting the large difference between the solvating powers of phenol and benzyl alcohol.
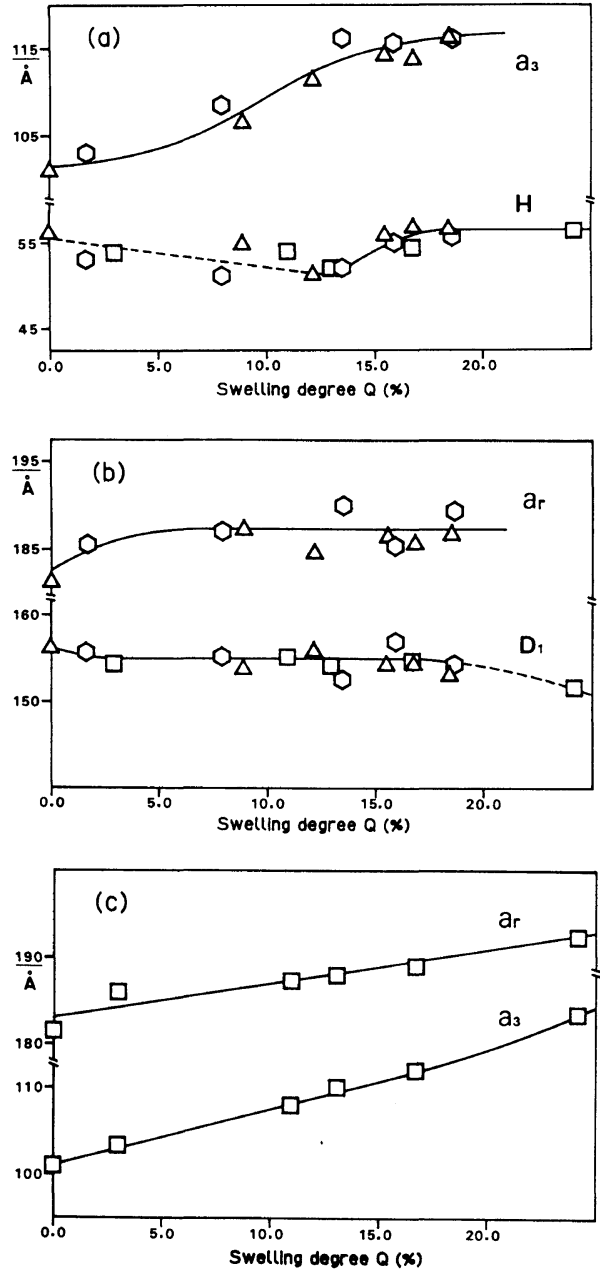

Figure 11. Dependence of the superstructure parameters of systems b $(\triangle), \mathrm{c}(\mathrm{O})$, and $\mathrm{d}(\square)$ (sample B) on the swelling degree $Q$. (a) $a_{3}$ and $H v s$. $Q$; (b) $a_{r}$ and $D_{1}$ vs. $Q ;$ (c) $a_{3}$ and $a_{r} v s$. $Q$.

The thickness of the swollen amorphous layer at maximum swelling degree for systems a and d was $63 \AA$ and $64 \AA$, respectively. On the other hand, the length of the tie molecules composed of 7 repeating units is about $62 \AA$, which agrees well with the above experimental values of the thickness of the amorphous layer derived from the SAXS experiment. This indicates that the tie molecules were highly stretched at high swelling degrees, presumably assuming extended conformation, although the 
actual situation may be more sophisticated because of the possible chain entanglement occuring in the amorphous region.

The peak widths, which are often related to the crystallite size and/or crystalline orders,${ }^{44}$ of section A and section B varied reversibly with the swelling degree. The present SAXS analysis showed, nevertheless, there are no well-defined correlations between this variation and any improvement of the structural perfectness, such as lamellar thickening or lateral order's improving. Only the variation of the long period may be responsible for the reversible behavior since the spread of a reciprocal point is proprotional to the distance from the origin. ${ }^{45}$ The plot in Figure 8 implies that the width of the peak is exclusively controlled by the long period in the present case because all the observed values were statistically on the same line. This is also predicted by the theoretical calculation using eq 7.

Acknowledgements. This work was supported in part by Grants-in-Aid for Scientific Research (No. 62470092) from the Ministry of Education, Science, and Culture of Japan. We would like to thank the staff of the Workshop for Experimentation and Practice, Faculty of Engineering, Nagoya University for manufacturing the cell for swelling for this work.

\section{REFERENCES}

1. M. Dosiere and J. J. Point, J. Polym. Sci., Polym. Phys. Ed., 22, 749 (1984).

2. M. Dosiere, J. Mater. Sci., 18, 1941 (1983).

3. Y. Udagawa and A. Keller, J. Polym. Sci. A-2, 9, 437 (1971).

4. G. V. Fraser, A. Keller, E. J. George, and D. Dreyfuss, J. Macromol. Sci.-Phys., B16, 295 (1979).

5. E. Ergoz and L. Mandelkern, J. Polym. Sci., Polym. Lett. Ed., 10, 631 (1972).

6. I. R. Harrison, T. J. Weaver, and J. Runt, Polym. Commun., 26, 244 (1985).

7. J. Descamps-Carlier, G. A. Homes, and J. J. Point, Makromol. Chem., 109, 1 (1967).

8. I. L. Hay and A. Keller, J. Mater. Sci., 2, 538 (1967).

9. A. Goffin, M. Dosiere, J. J. Point, and M. Gilliot, J.
Polym. Sci. C, 38, 135 (1972).

10. F. Kloos, S. Go, and L. Mandelkern, J. Polym. Sci., Polym. Lett. Ed., 17, 161 (1979).

11. K. Göttlicher, W. Fronk, and W. Wilke, Coll. \& Polym. Sci., 261, 126 (1983).

12. W. Fronk and W. Wilke, Coll. \& Polym. Sci., 263, 97 (1985).

13. W. Fronk and W. Wilke, J. Polym. Sci., Polym. Phys. Ed., 24, 839 (1986).

14. C. E. Rogers, V. Stannett, and M. Szwarc, J. Phys. Chem., 63, 1406 (1959).

15. K. P. Kwei and T. K. Kwei, J. Phys. Chem., 66, 2146 (1962).

16. D. A. Blackadder and J. S. Keniry, J. Appl. Polym. Sci., 16, 1261 (1972).

17. N. F. Brockmeier, R. W. McCoy, and J. A. Meyer, Macromolecules, 6, 176 (1973).

18. H. R. Brown, J. Polym. Sci., Polym. Phys. Ed., 16, 1887 (1978)

19. W. R. Parrish, J. Appl. Polym. Sci., 26, 2279 (1981).

20. P. J. Flory and J. Rehner, J. Chem. Phys., 11, 512 (1943).

21. J. Rubin and R. D. Andrews, Polym. Eng. Sci., 8, 302 (1968).

22. K. Hoashi and R. D. Andrews, J. Polym. Sci., C, 38, 387 (1972).

23. K. Inoue and S. Hoshino, J. Polym. Sci., Polym. Phys. Ed., 15, 1363 (1977).

24. S. Ogasawara, M. Ishiyama, and S. Kuroiwa, Sen'i Gakkaishi, 34, T232 (1978).

25. G. Hinrichsen and H. Wick, Melliand Textilber./Int. Text. Rep. (Ger. Ed.), 60, 182 (1979).

26. D. R. Subramanian, A. Venkataraman, and N. V. Bhat, J. Macromol. Sci.-Phys., B18, 177 (1980).

27. R. A. F. Moore, H. D. Weigmann, and C. A. Morello, Book Pap., Natl. Tech. Conf.-AATCC, 109 (1981).

28. D. R. Subramanian, A. Venkataraman, and N. V. Bhat, Man-Made Text. India, 24, 39 (1981).

29. W. C. Ingamells, J. Soc. Dyers Colour, 96, 466 (1980).

30. D. R. Subramanian, A. Venkataraman, and N. V. Bhat, J. Appl. Polym. Sci., 27, 4149 (1982).

31. S. Malei-Kveder and F. Kocevar, Melliand Textilber./Int. Text. Rep. (Ger. Ed.), 63, 681 (1982).

32. T. Wakida, Y. J. Lim, T. Takagishi, N. Kuroki, and S. C. Choi, Sen'i Gakkaishi, 39, T535 (1983).

33. T. K. Kwei and H. M. Zupko, J. Polym. Sci. A-2, 7, 867 (1969).

34. G. W. Miller, S. A. D. Visser, and A. S. Morecroft, Polym. Eng. Sci., 11, 73 (1971).

35. W. V. Titow, M. Braden, B. R. Currell, and R. J. Loneragan, J. Appl. Polym. Sci., 18, 867 (1974).

36. R. Hosemann and S. N. Bagchi, "Direct Analysis of Diffraction by Matter," North-Holland, Amsterdam, 1962.

37. W. Wilke, Acta Cryst., A39, 864 (1983). 
38. N. F. Brockmeier, R. W. McCoy, and J. A. Meyer, Macromolecules, 5, 464 (1972).

39. D. Patterson, Y. B. Tewari, H. P. Schreiber, and J. E. Guillet, Macromolecules, 4, 356 (1971).

40. R. D. Newman and J. M. Prausnitz, J. Phys. Chem., 76, 1492 (1972).

41. E. L. V. Lewis and I. M. Ward, J. Macromol. Sci.Phys., B18, 1 (1980).
42. R. Huisman and H. M. Heuvel, J. Polym. Sci., Polym. Phys. Ed., 14, 941 (1976).

43. H. M. Heuvel, R. Huisman, and K. C. J. B. Lind, $J$. Polym. Sci., Polym. Phys. Ed., 14, 921 (1976).

44. R. J. Matyi and B. Crist, Jr., J. Polym. Sci., Polym. Phys. Ed., 16, 1329 (1978).

45. S. Arnott, Polymer, 6, 478 (1965). 